\title{
The Design of Vehicle Tire Pressure Monitoring System Based on Bluetooth
}

\author{
Liqing Geng ${ }^{1, a^{*}}$, Li Zhao ${ }^{2, b}$, Zeyu Zhang ${ }^{3, c}$ \\ ${ }^{1}$ Tianjin Key Laboratory of Information Sensing and Intelligent Control, Tianjin University of \\ Technology and Education, Tianjin, 300222, China \\ 2 Tianjin University of Technology and Education, Tianjin, 300222, China \\ ${ }^{3}$ Tianjin University of Technology and Education, Tianjin, 300222, China \\ agenglq2004@126.com, bjinshihui@163.com, czhangzeyu1335@sina.cn
}

\begin{abstract}
Keywords: Tire pressure; Bluetooth; Monitoring
Abstract. A car backfiring is an important cause of traffic accidents. The vehicle tire pressure monitoring system (TPMS) is an automobile accident prevention system. This paper designs a tire pressure monitoring system of motor vehicles based on Bluetooth using the detection and sensing technology, single-chip microcomputer's control technology and Bluetooth technology synthetically. The system includes two parts of lower computer and upper computer (mobile phone) and the lower computer can monitor in real time and process the tire pressure parameters. In addition, the lower computer will send the tire pressure data processed by it to the mobile phone through Bluetooth, and then mobile phone checks the data and displays the detecting results to inform the driver of the state of vehicle tire pressure. This system realizes some functions including the detection of tire pressure in real time, display of tire pressure data and warning function through mobile application.
\end{abstract}

\section{Introduction}

With the development of automobile industry in recent years, traffic becomes more and more convenient, thus traffic accidents have also increased significantly among those accidents, because the accident rate generated by tire's abnormal condition is also high, drivers have to check that the car tire pressure is within a safe range or not at any time while driving a car. The research from China's Traffic Safety Committee points out the important preventing measures of car backfiring include that keeping the vehicle's tire pressure within the safe range effectively and becoming aware of the damaged tire as soon as possible. Obviously the vehicle tire pressure monitoring system must will become the primary safety precautions. Through the security assurance of vehicle tire pressure monitoring system, we can decrease the quantity of traffic accidents to guarantee the car's safe driving[1].

This paper studies a wireless vehicle tire pressure monitoring system based on the communication technology of Bluetooth, and the system can collect in real time and send the information of tire pressure to inform this car's driver of tire pressure in the first place, moreover, when tire pressure is beyond normal and safe range, the driver of this car, related personnel out of this car and driver of nearby car can receive the warning signal and take effective emergency measures to restrain traffic problems efficiently.

\section{The Design of System Schematic}

The overall block diagram of system is shown in the Fig.1. This system consists of lower computer's control unit and receiving terminal of smart phone. Lower computer's control unit collects tire pressure data in real time and sends it to the smart phone's receiving terminal by Bluetooth. The lower computer module and smart phone will judge whether the tire pressure data is within the safe range, and if tire pressure data is beyond the safe range, smart phone will vibrate and its operation interface 
will show that the tire pressure is abnormal. In addition, the warning lights on the car's maneuvering desk will shine and the buzzer out of car will generate alarm signal.

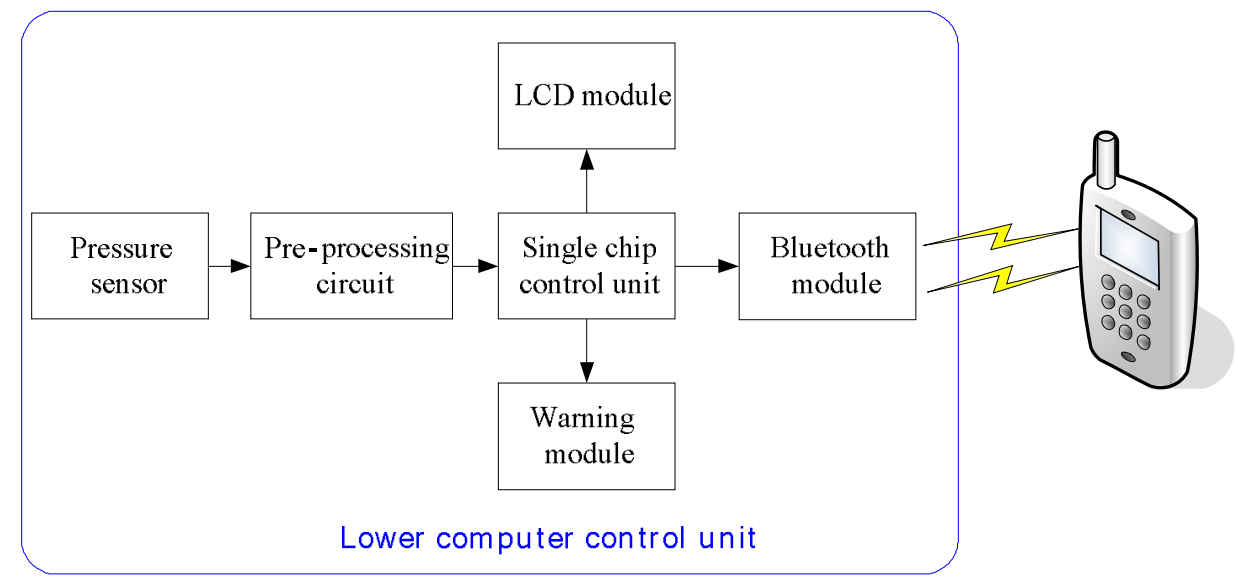

Fig.1 The block diagram of system design

\section{The Design of Hardware Circuit}

The system selects STC12C5A60S2 MCU as the control core and XGZB 7-Piezo resistive pressure sensor to collect tire pressure signal, and LCD1602 displays tire pressure value in real time. Moreover, the system uses HC-07 Bluetooth module to send data to mobile terminal.

STC12C5A60S2[2] is High speed/low power/super anti-interference 8051 series single chip microcomputer. It is fully compatible with the traditional instruction code of 8051, it's running speed is faster 8 to 12 times than the former. MAX810 special reset circuit , 2 road PWM and 8 high-speed road 10 bit A/D conversion $(250 \mathrm{k} / \mathrm{S})$ are integrated inside the chip. So it is suitable for automobile tire pressure monitoring system.

\section{The Design of Sensor's Circuit}

The sensor's circuit is shown in Fig.2. The first feet of XGZB7 sensor[3] is connected to ground, and the fourth feet is connected to the $+5 \mathrm{~V}$ power provided by power adapter to power the sensor. The second and third feet are short circuit and then connected to positive end of the differential operational amplifier circuit. The six feet is connected to negative end of the differential operational amplifier circuit. The sensor can convert the $0 \sim 1000 \mathrm{kPa}$ pressure into $0 \sim 100 \mathrm{mv}$ voltage signal which then will be transmitted to differential subtraction operational circuit.

\section{The Design of Pre-processing Circuit}

Pre-processing circuit is shown in Fig.3. The differential subtraction operation circuit amplifies the voltage signal inputted by sensor from $0 \sim 100 \mathrm{mV}$ to $0 \sim 5 \mathrm{~V}$, then the signal is transmitted to the signal-inputting terminal P1.0 of single chip microcomputer. 


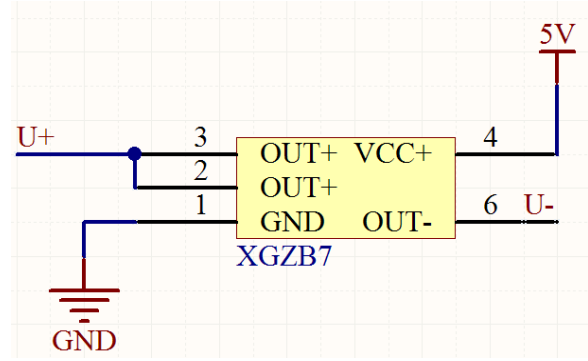

Fig.2 The sensor circuit diagram

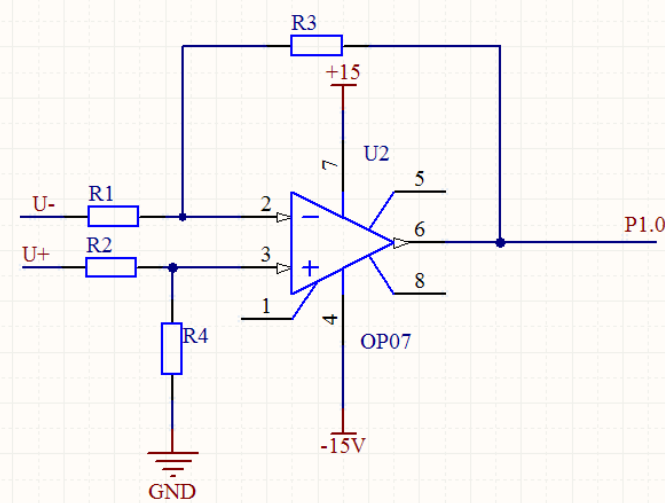

Fig.3 The pre-processing circuit

\section{The Design of LCD Display Circuit}

The LCD display circuit is shown in Fig.4. The variable resistors in the figure is used to adjust bias signal of the LCD.

\section{The Design of Bluetooth Transmitter Circuit}

HC-07 Bluetooth[4] transmitter circuit diagram is shown in Fig.5. Microcontroller's P3.0 is connected to TXD of Bluetooth transmitter circuit, and Bluetooth module's pin 2 is connected to $+5 \mathrm{~V}$ power supply of VCC. Moreover, Bluetooth module's pin 3 is connected to $0 \mathrm{~V}$ power supply of GND.

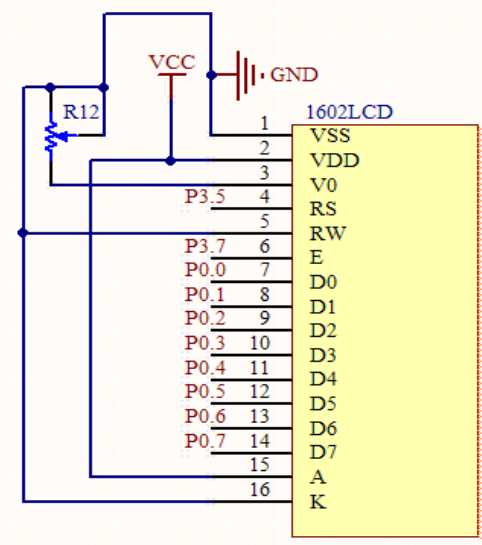

Fig.4 The LCD display circuit diagram

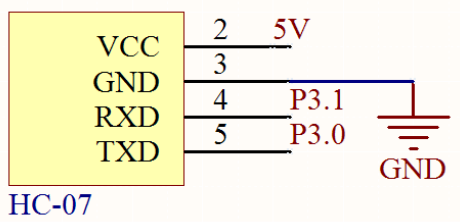

Fig.5 Bluetooth transmitter circuit diagram

\section{The Design of Software}

\section{The Design of Lower Computer Software}

The lower computer software mainly realizes some functions including collection, management and warning of the tire pressure data. The lower computer's software was composed of the main program, data collection subprogram, LCD display subprogram and Bluetooth communication subprogram. The main program is shown in Fig.6. Firstly, the system will initializes STC12C5A60S2 microcontroller, 1602 LCD screen and Bluetooth serial port configuration. Then the system collects pressure data in 
real time, displays the data on LCD screen and sends data to Bluetooth module at the same time. In addition, the system will generate the sound and light alarm if the collected tire pressure data exceeds the value of the safe range.

\section{The design of mobile terminal software}

The smart phone's development platforms of upper computer mainly include Android, Windows Mobile and Symbian etc., among them, the Android platform[5] is open and its development and application operation are convenient. Besides its compatibility is better than other platforms. To sum up, Android platform is very suitable for the development of upper computer module in automobile tire pressure monitoring technology. This system uses the XML file configuration to design user's interface. User's interface of vehicle tire pressure monitoring system in upper computer is shown in Fig.7. In the figure, the abscissa of coordinate axis is used to represent time in order to dynamically show the exact time when the system collect automobile tire pressure value, and ordinate axis is used to represent detected tire pressure value. The interface displays automobile tire pressure value's curve in real time to observe system's monitoring state. The exact design methods is as following:

Due to the data increases gradually, fixed-length arrays can't satisfy the need of display, so this system uses two dynamic arrays to add new data and has achieved prospective display effect.

Drawing a punctuation on display interface needs to invoke two dynamic arrays to add data, and then the system uses plotting program provided by android and according logic to draw the punctuation. Automobile tire pressure graph adopts line chart and android drawing API and defines two dynamic arrays, and one array is used to deposit temperature value and another deposit time when the system receives data. The unit value of the ordinate axis's every scale is constant because automobile tire pressure value is within a definite numerical range. In addition, the abscissa axis shows time when the system receives data and every time interval is also equal. Time interval of two adjacent arrays is the time interval that single-chip microcontroller sends data. When the new data are received and collected to the dynamic array, android drawing API will draw again to realize the display of automobile tire pressure data's dynamic curve.
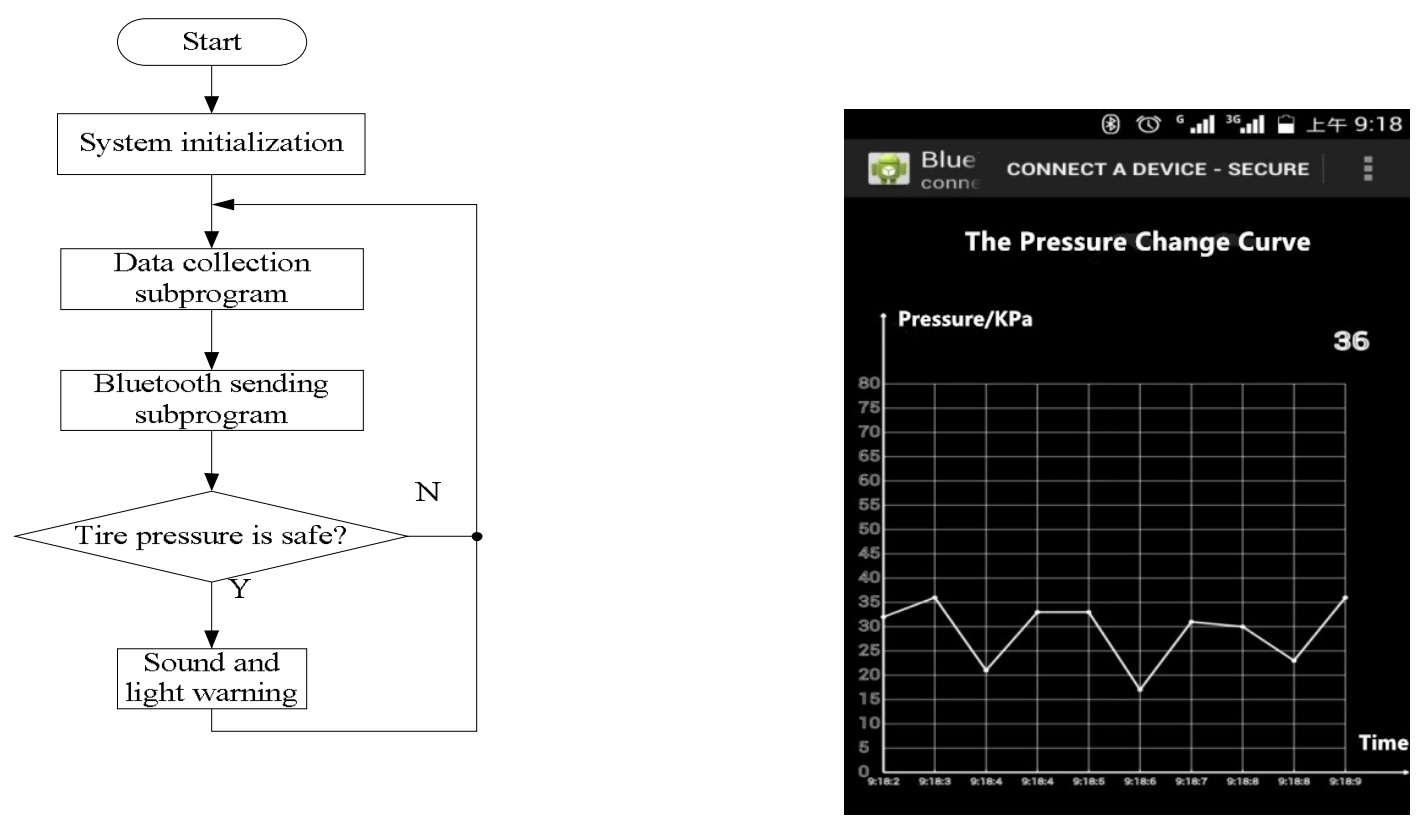

Fig.6 The main program flow chart

Fig.7 The mobile monitoring interface 


\section{Conclusions}

This paper designs a automobile tire pressure monitoring system based on Bluetooth technology, and the system includes two parts of lower computer and upper computer (mobile phone) and the lower computer can monitor in real time and process the tire pressure parameters. In addition, it will send the tire pressure data processed by lower computer to the mobile phone through Bluetooth, and then mobile phone checks the data and displays the detecting results to inform the driver of the state of vehicle tire pressure. This system realizes some functions including the detection of tire pressure in real time, display of tire pressure data and warning function through mobile application. The system's debugging result show that this system operates reliably and it satisfies the required functions and achieves the prospective technical indexes.

\section{References}

[1] YU Xiu-wu, YANG Xian-yang, MA Ru-cheng, WANG Yun-xing, YU Yuan-qin, ’Design and implementation of automobile safety tire pressure monitoring system based on SP12," Journal of Safety Science and Technology, 2012(8). (in Chinese)

[2] HONG Zhi-gang, DU Wei-ling, "Single chip microcomputer application system design," Mechanical industry press,2011. (in Chinese)

[3] WU Jian-ping, "The principle and application of sensor," Mechanical industry press,2012. (in Chinese)

[4] HE Guo-quan,. Design of wireless access point based on bluetooth. Microcomputer \& Its Applications.2010 (18). (in Chinese)

[5] WU Ya-feng, SUO Yi-na, "The core technology and the example explanation," Electronic industry press,2010. (in Chinese)

\section{Acknowledgments}

Fund program: Natural Science Foundation of China (61178081). Natural Science Foundation of Tianjin (14jczdjc3630). Beforehand research project for schools (jk14-13). 